International Journal of Engineering \& Technology, $7(1.2)(2018) 58-60$
International Journal of Engineering \& Technology
WPC
Website: www.sciencepubco.com/index.php/IJET
Research paper

\title{
A review: algorithm used for beam forming systems
}

\author{
Soma Pal *, Azazul Haque \\ Department of Electronics of Communication Engineering Vel Tech Rangarajan Dr. Sagunthala $R$ \& D \\ Institute of Science and Technology, Avadi, Chennai- 600062. India \\ *Corresponding author E-mail:spg2716@gmail.com
}

\begin{abstract}
Wireless communication uses a smart antenna to provide better coverage and capacity for the communication system. Main functions performed by the smart antenna are Direction of Arrival estimation (DOA) and beamforming (DBF). The beam forming is signal processing techniques which combine antenna array technology with high-performance up/down-conversion, analog to digital conversion and digital signal processing to provide receivers with very high spatial selectivity. This paper evaluates non-blind algorithm such as LMS, to compute the weight calculation for phased array antenna using Matlab Simulink.
\end{abstract}

Keywords: DOA; Beam forming; LMS and Weight calculation.

\section{Introduction}

A smart antenna can be defined as an array of antenna elements connected to a digital signal processor. In order to calculate a direction of arrival of a signal using a signal impression, an antenna array is implemented using signal processing algorithm. The implementation of the smart antenna must not be confused with the reconfigurable antenna as single element antennas can be treated as smart antenna whereas a reconfigurable antenna contains an array of antenna. A smart antenna mainly performs two functions as DOA estimation and Beam forming.

$\mathrm{DBF}$ is a signal processing technique used for transmission and reception of the signals to establish maximum spatial selectivity. The concept of beamforming is to point the antenna array and shape the beam by giving each antenna the right delay and weight, and then adding the signals. In the broad sense, beamforming techniques are classified as Conventional beam formers and Adaptive beam formers or Phased array.

In this paper, we focus on the studying the architecture of phased array beam formers which can track the angle of arrival of RF signals and are utilized predominantly in digital receivers where the RF signals received are detected and digitized. The RF signal received from an antenna element is down-converted using digital down conversion (DDC). And the LMS algorithm must process the baseband signal where each antenna element has its own ADC and DDC channels.

\section{DSB receiver}

Beam forming performance mainly depends on how the receiver is designed. This receiver architecture has N ADC's and N DDC's for $\mathrm{N}$ antenna elements. A digital beam-forming receiver system sums weighted versions of every receiver output to form antenna patterns. Fig.1 illustrates a simple block diagram of conventional DBF receiver system [1] [2] [3].

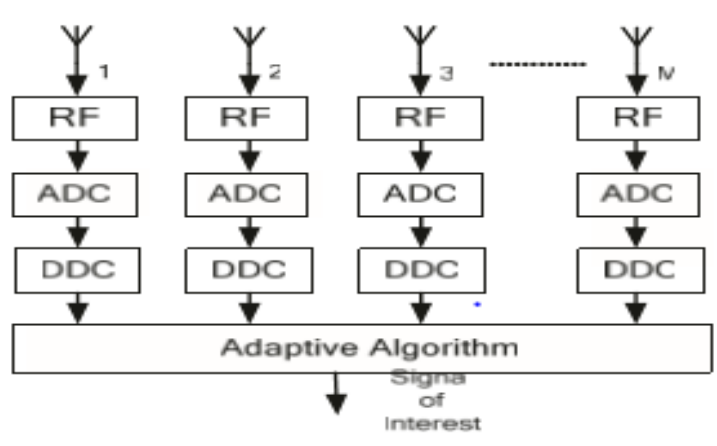

Fig. 1: Block Diagram of DBF Receiver System.

\section{Adaptive beamforming algorithms}

Adaptive Beam forming is the process of recursively updating the complex weights for each of the antenna elements used in the array to achieve maximum reception in desired direction. The algorithm is used for optimization of weight adaption of sensor array; hence this technique is named as adaptive beam forming technique.

The concept of adaptive beamforming algorithm is to rejects all signals of same frequency coming from other direction than the desired Direction of arrival (DOA) - this singular fact helps to reduce co-channel interference. The Adaptive DBF receiver also performs a selective filtering of arriving signals. In early 1960, the adaptive beam forming technique was developed for military applications using sonar and radar. However, with the advancement, it was extended to several biomedical ultrasonic imaging and seismic applications where various beamforming techniques were found suitable.

For a beamformer with constant weights does not track the signal environment; the resulting because of beam pattern. However, adaptive array may change its pattern automatically in response to the signal environment [4]. 


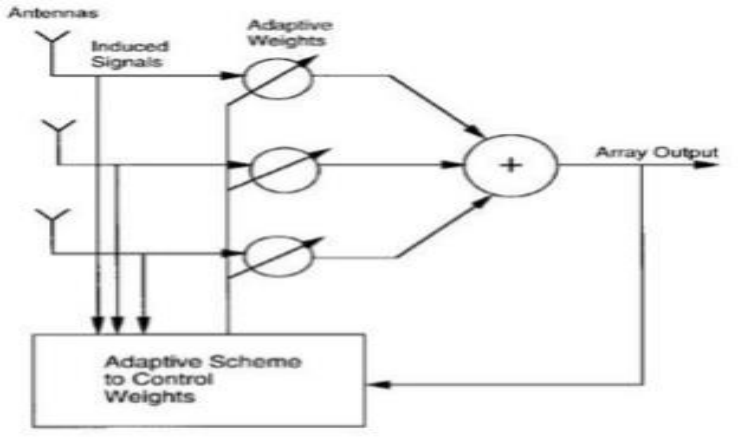

Fig. 2: Adaptive Scheme.

\subsection{LMS algorithm}

In 1960 this algorithm was first developed by Widrowand Hoff and later the design of this algorithm was stimulated by the Wiener - Hopf equation. Using stochastic gradient approach , a set of Wiener - Hopf equations were modified and a simple adaptive algorithm that can be updated recursively was developed ; which was later on named as the least-mean-square (LMS) algorithm. The algorithm contains three steps in each recursion: the computation of the processed signal with the current set of weights, the generation of the error between the processed signal and the desired signal, and the adjustment of the weights with the new error information. LMS (least mean square) algorithm is used because of its simplicity, small calculations and higher stability [5] [6] [7]. Compared to recursive least squares (RLS) algorithms, the LMS algorithms do not involve any matrix operations, and also it requires fewer computational resources and memory compared to the RLS algorithms which makes it less complicated. However, the Eigen value spread of the input correlation matrix, or the correlation matrix of the input signal, might affect the convergence speed of the resulting adaptive filter.

\subsection{LMS adpative filter}

An adaptive filter is a circuit which computes iteratively; represents the relationship between the input and output signals of a filter. And the filter coefficients are self-adjusted according to an adaptive algorithm used. The diagram of a typical adaptive filter is shown in Figure 3.

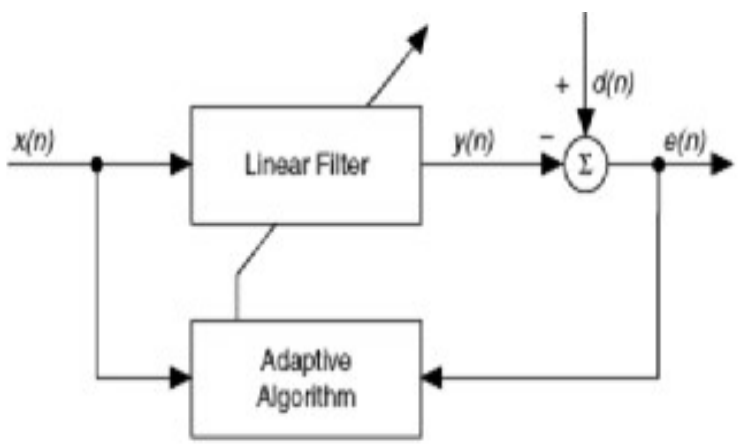

Fig. 3: LMS Adaptive Filter.

Where, $x(n)$ is the input signal to a linear filter; $y(n)$ is the corresponding output signal; $d(n)$ is an additional input signal to the adaptive filter; e(n) is the error signal that denotes the difference between $d(n)$ and $y(n)$.

The linear filter can use different filter types such as finite impulse response or infinite impulse response whereas an adaptive algorithm is used to adjusts the coefficients of the linear filter iteratively to minimize the power of e (n).

\subsection{ADC realization in simulink}

We know that analog-to-digital converter (ADC, A/D, A-D, or Ato-D) is a system that converts an analog signal, such as a sound picked up by a microphone or light entering a digital camera for example, into a digital signal. An ADC may also provide an isolated measurement such as an electronic device that converts an input analog voltage or current to a digital number proportional to the magnitude of the voltage or current. Typically the digital output is a two's complement binary number that is proportional to the input, but there are other possibilities. There are several ADC architectures available. Due to the complexity and the need for precisely matched components, most specialized ADCs are implemented as integrated circuits (ICs). A digital-to-analog converter (DAC) performs the reverse function; it converts a digital signal into an analog signal. In figure 4 shows the implementation of ADC using Simulink.

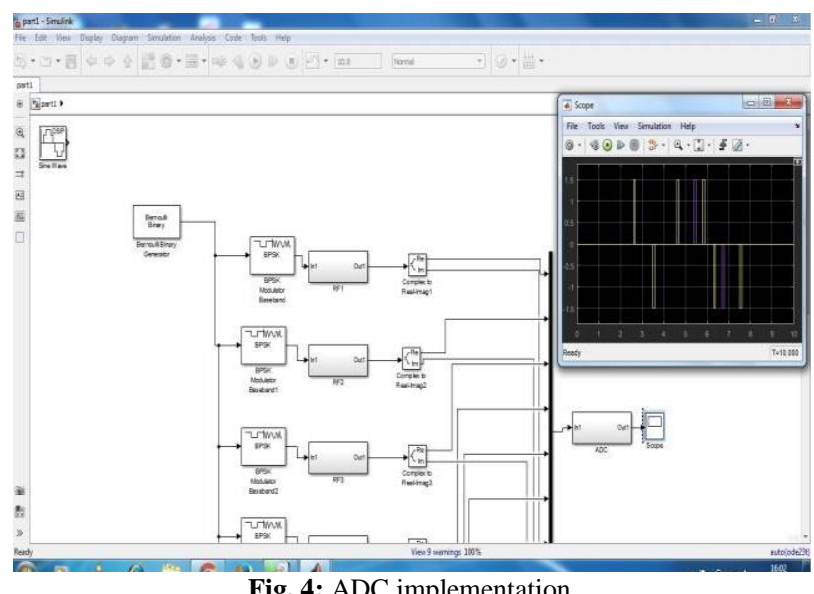

\subsection{Hardware realization in simulink}

The DBF receiver system consists of uniformly spaced linear 8 antenna elements where the distance between adjacent antennas is kept a half wavelength ' $\lambda / 2$ ' with a $3 \mathrm{GHz}$ carrier frequency of the system. The received radio signals are digitized using both a 32-bit idealized ADC and then down-converted to $30 \mathrm{MHz}$. The Real and complex signals generated from the digitally down converter separately passed on to the LMS algorithm that updates the complex weights.

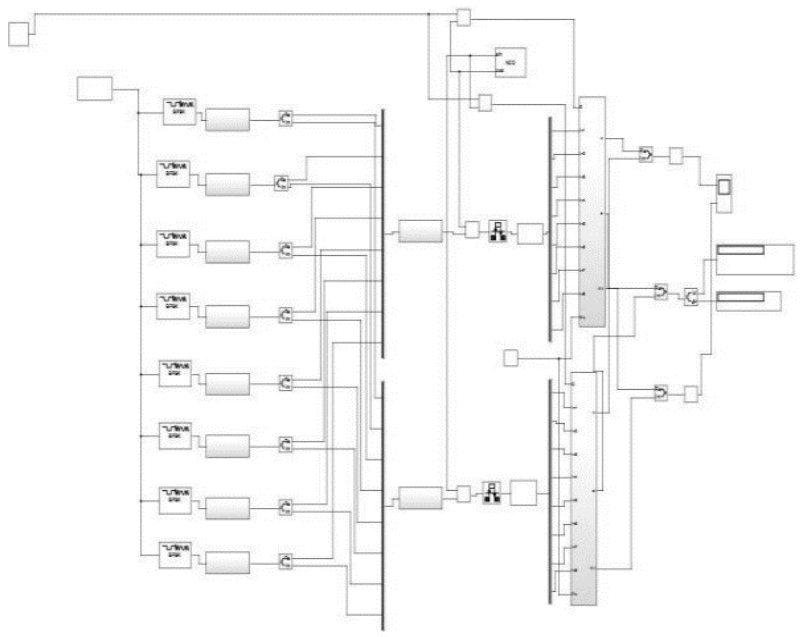

Fig. 5: Implementation of DBF Receiver.

\subsection{Null placement}

Null steering is applied to suppress the unwanted signals in some specific direction. The Null is placed in the angles like 3060 and 100. So that there will be no beam formation in those particular angles. Null Placement is shown in the below Figure 6. 

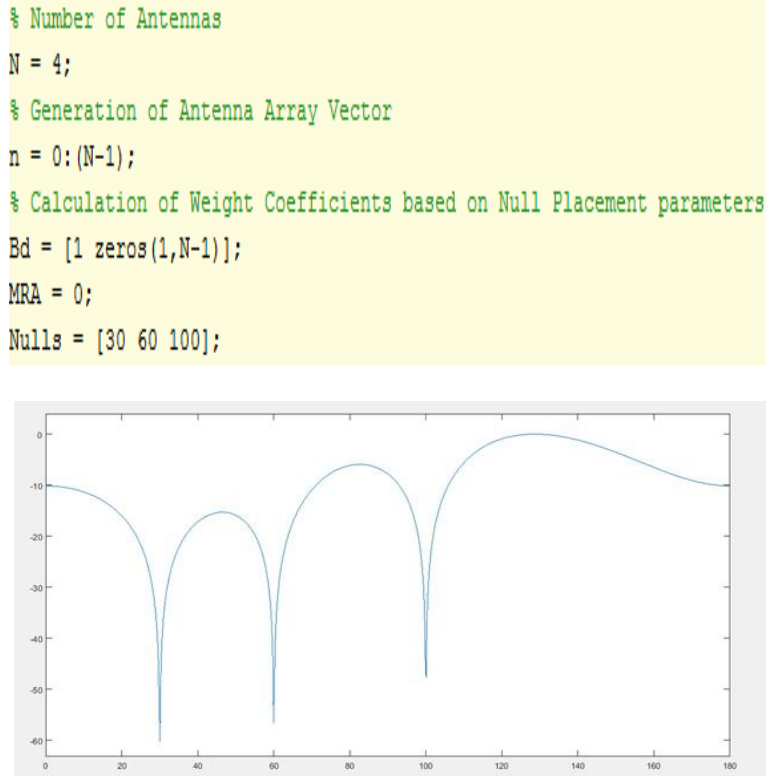

Fig. 6: Null Placed at $30^{\circ}, 60^{\circ}$ and 100 .

\section{Conclusion}

The LMS algorithm based adaptive beamforming weights converges within 200 samples for the each step- size $\mu=0.005$. The performance of the antenna gets better when the number of antenna elements increases in a receiver. It is observed that LMS algorithm is converging after 50 iterations. The performance of the LMS algorithm is compared with the effect of varying the number of antenna elements and distance between array element on the array factor and Mean Square Error (MSE). Overall MSE tends to be almost the same for the given values of an antenna element and then MSE gives an optimum error in the desired direction.

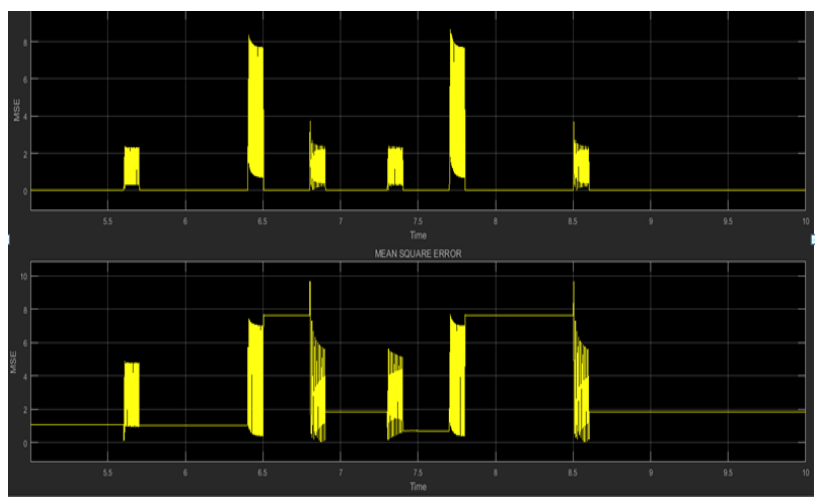

Fig. 7: Mean Square Error of LMS Algorithm.

\section{References}

[1] Mohammad Salman Baig, B. Ramaswam Karthikeyan, "Improved Receiver Architecture for Digital Beamforming Systems" International Conference on Computer, Communication and Electrical Technology, 2011 .

[2] Miss Nayan B. Shambharkar, Prof. Prakash M. Mainkar, Dr. G. N. Mulay, "Implementation of RLS Beamforming Algorithm for Smart Antenna System" International Journal of Engineering and Management Research - April 2014, Volume-4, Issue-2.

[3] Revati Joshi, Ashwini Kumar Dhande, "Adaptive Beamforming Using Lms Algorithm” International Journal of Research in Engineering and Technology. 2014. ISSN: 2319-1163.

[4] P. Kabilan, K Meena Alias Jeyanthi, "Performance Comparison of A Modified LMS Algorithm In Digital Beam Forming For High Speed Networks" ICCIMA, 2007.

[5] Jalal Abdulsayed Srar, Kah-Seng Chung and Ali Mansour, "Adaptive Array Beamforming using a Combined LMS-LMS Algorithm", Aerospace Conference, IEEE, 2010.
[6] Vijendra Mishral, Gaurav Chaitanya, "Analysis of LMS, RLS and SMI Algorithm on the Basis of Physical Parameters for Smart Antenna, IEEE.

[7] Constantine A.Balanis, 'Antenna Theory Analysis and Design,' 3/e 2005. 